\title{
Undercut-Proof Subgame Perfect Equilibrium of a Pricing Game
}

Roy, Jaideep; Datta, Debabrata

Publication date:

2001

Document version

Early version, also known as pre-print

Citation for published version (APA):

Roy, J., \& Datta, D. (2001). Undercut-Proof Subgame Perfect Equilibrium of a Pricing Game. Department of Economics, University of Copenhagen. 


\title{
DISCUSSION PAPERS Department of Economics University of Copenhagen
}

$$
\text { 01-04 }
$$

Undercut-proof Subgame Perfect Equilibrium of a Pricing Game

\author{
Debabrata Datta \\ J aideep Roy
}

Studiestræde 6, DK-1455 Copenhagen K., Denmark Tel. +45 35323082 - Fax +45 35323000 http://www.econ.ku.dk 


\title{
Undercut-proof subgame perfect equilibrium of a pricing game
}

\author{
Debabrata Datta*
}

Department of Economics

Asutosh College, Calcutta University

92 S. P. Mukherjee Road, Calcutta 700 026, India

\author{
Jaideep Roy ${ }^{\dagger}$ \\ Institute of Economics \\ University of Copenhagen \\ Studiestræde 6 \\ DK 1455 Copenhagen K, Denmark
}

April 10, 2001

\begin{abstract}
Two firms selling a homogenous product to two types of buyers are involved in a sequential pricing game with zero costs. The pricing strategy available involves a fixed price and a royalty. It is shown that there exists a unique subgame perfect equilibrium with positive profits to both firms if and only if buyers differ significantly in their willingness to pay. In particular, the incumbent sets a positive royalty and sells to the low demand buyer while the entrant only charges a fixed price and sells to the high demand buyer, resulting in an undercut-proof subgame perfect equilibrium.

KEYWORDS: sequential pricing, fixed price, royalty, undercut-proof subgame perfect equilibrium situation.

JEL: C73, D43, L13.

\footnotetext{
* Debabrata Datta gratefully acknowledges research support and hospitality provided by the Institute of Economics, University of Copenhagen during the preparation of this manuscript, and thanks Birgit Grodal in particular in this regard. tel: +91 33485 2490; e-mail: datta_debabrata@mantraonline.com

†tel: $+45 \overline{35} 324422$; fax: +45353230 00; e-mail: jaideep.roy@econ.ku.dk
} 


\section{Introduction}

In the late seventies, Xerox came to India to sell photocopying machines and as has been their usual practice throughout the world, they introduced a pricing policy which charged a fixed price per machine and a royalty on each copy made. Within a couple of years, Canon also entered the market with a pricing policy where such royalties were absent. With the advent of the photocopying technology, many individuals started setting up small businesses which involved photocopying for others against a price per copy. Depending upon the location of the copying shop, such enterprises could be classified into high and low demand local monopolists. It so happened that over these years most of the low demand users preferred Xerox while Canon became popular with the high demand ones. Why did Canon not opt for a pricing policy exactly like that of Xerox and charge a fixed price a little less than Xerox as then Canon would have taken the entire market of such shopkeepers, like in a typical price competition model with homogenous product?

To address this issue, we use a model with two locally monopolistic shopkeepers who differ in the demand they face for photocopying and each shopkeeper buys only one machine. There are two firms who sell these machines and both have the option of charging a royalty or not. Between them, one firm is an incumbent while the other is a potential entrant. This is a typical sequential pricing game with the qualification that the pricing technology is some form of a 'tie-in' (see Tirole 1994, pp 146). ${ }^{1}$ Firms have zero cost and thus since undercutting is feasible, the incumbent gets zero profits unless it can induce the entrant, by a suitable choice of strategy, not to undercut over the existing price. We call such a possibility (if it exists) undercut-proofness. Buyers choose to buy from the firm whose pricing technology is more attractive while whenever they are indifferent, they always buy from the entrant. ${ }^{2}$ We show that there exists a unique undercut-proof

\footnotetext{
${ }^{1}$ As an example of another type of pricing technology, Blackstone (1975) studies a copying machine industry where an incumbent monopolist (SCM) used tie-in policy by forcing the customers to use a special type of paper which was produced by the firm itself.

${ }^{2}$ This is a tie-breaking simplification with the interpretation that if the entrant offers
} 
subgame perfect equilibrium where

(i) both firms can earn positive payoffs under our general pricing-strategy space if and only if shopkeepers differ significantly in the size of demand they face individually,

(ii) the incumbent firm always charges a fixed price and a positive royalty per copy while the entrant only uses a fixed price,

(iii) the pricing contract offered by the incumbent uses a royalty which is strictly less than that if it were a monopolist and decided to serve both the shopkeepers, and

(iv) the low demand shopkeeper buys from the incumbent while the high demand one buys from the entrant.

\subsection{Related literature}

There is a large literature that studies price competition since Bertrand's (1883) critique against Cournot conjectures. In general it is shown that linear price competition drives profits to competitive levels due to the threat of price undercutting, excepting when firms face capacity constraints (Kreps and Scheinkman (1983), or the goods are differentiated.

With the possibility of non-linear pricing, vertical market segmentation results when consumers differ in their valuation of the product. Spence (1977) shows that there exists a non-linear pricing strategy that divides the market on the basis of different amounts purchased by individual consumers. A similar phenomenon occurs in our case as well. Mussa and Rosen (1978) finds similar separating equilibria assuming variation in the quality of the product purchased.

Wallner (1999) analyzes a finite horizon sequential move pricing duopoly with a homogenous product, where firms use Markov strategies and earn payoffs each period. This is in contrast to our case where an extensive form game is studied and payoffs are realized only when all players have chosen

a price such that the a buyer is indifferent between buying from the incumbent and the entrant, the entrant actually undercuts infinitesimally. 
their strategies. The solution yields stationary patterns where the reaction functions follow 3-period cycles. The market price never settles down and is at all times strictly above marginal costs.

Closest to our work is the one by Morgan and Shy (1996). They propose an equilibrium concept similar to ours called Undercut-Proof equilibrium in a single-shot pricing game between firms producing differentiated brands and show that whereas a Nash-Bertrand equilibrium in pure actions never exists, a unique undercut-proof equilibrium always exists and brand prices monotonically diverge when the brands become more differentiated and are identical when the brands become homogeneous. However in our model, a similar equilibrium is derived even with a homogenous good but with variation in the valuation of buyers. We also observe a similar monotonic divergence pattern driven by different demand intercepts of the shopkeepers.

The rest of the paper is structured as follows. In section 2 we describe the model. Section 3 addresses issues regarding existence, non-existence and uniqueness of subgame perfect equilibria of the game induced by the environment studied. Finally the paper concludes in section 4 .

\section{The model}

Two firms, $x$ and $y$, wish to sell a homogenous good which can be used as an input to generate profits. For simplicity and without any loss of generality we assume zero cost of production of the good. The good is purchased by two shopkeepers, 1 and 2. The good can be used by the shopkeepers to produce output which can be sold in a market. The shopkeepers are local monopolists and face inverse demand functions $p_{1}\left(q_{1}\right)=a-q_{1}$ and $p_{2}\left(q_{2}\right)=1-q_{2}$ respectively. We assume for simplicity that once the shopkeepers acquire the good, they face zero costs of production. Let $\left(\pi_{i}, A_{i}\right) \in \mathbb{R}_{+} \times \mathbb{R}_{+}$be the price contract offered by firm $i=x, y$. If shopkeeper $j=1,2$ buys the good from firm $i$ and decides to sell $q_{j}$ units of his product, then the payoff to shopkeeper $j$ is

$$
u_{j}\left(\pi_{i}, A_{i}, q_{j}\right)=p_{j}\left(q_{j}\right) q_{j}-\pi_{i} q_{j}-A_{i}
$$


while the payoff to firm $i$ is

$$
U_{i}\left(\pi_{i}, A_{i}, q_{j}\right)=\pi_{i} q_{j}+A_{i} .
$$

Firm $x$ is the 'leader' and is the first to offer $\left(\pi_{x}, A_{x}\right)$. After observing the move of $x$, firm $y$ offers $\left(\pi_{y}, A_{y}\right)$. The game ends and the shopkeepers self select themselves in their most preferred pricing option. All players have a zero reservation payoff.

In what we follows we are interested in finding the set of subgame perfect equilibria of the extensive form game induced by the above environment and played between the firms and the shopkeepers.

\section{Analysis}

Let us first consider the problem faced by shopkeeper $j$ who buys from firm $i$. Shopkeeper $j$ chooses $q_{j}$ to solve

$$
\operatorname{Maxp}_{q_{j}}\left(q_{j}\right) q_{j}-\pi_{i} q_{j}-A_{i} .
$$

The first order condition of the above problem yields optimal choice of $q_{j}$ equal to

$$
q_{j}^{*}\left(\pi_{i}, A_{i}\right)=\left\{\begin{array}{l}
\frac{a-\pi_{i}}{2} \text { for } j=1 \\
\frac{1-\pi_{i}}{2} \text { for } j=2
\end{array}\right.
$$

while the maximized payoff to shopkeeper $j$ is

$$
u_{j}^{*}\left(\pi_{i}, A_{i}\right)= \begin{cases}\frac{1}{4}\left(a^{2}-2 a \pi_{i}+\pi_{i}^{2}-4 A_{i}\right) & \text { for } j=1 \\ \frac{1}{4}\left(1-2 \pi_{i}+\pi_{i}^{2}-4 A_{i}\right) & \text { for } j=2\end{cases}
$$

Lemma 1 For any given $K \geq 0$, the loci $u_{1}^{*}\left(\pi_{i}, A_{i}\right)=K$ and $u_{2}^{*}\left(\pi_{i}, A_{i}\right)=$ $K$ are convex to $(0,0)$. Furthermore, the iso-payoff functions satisfy the single crossing property in the $\left(A_{i}-\pi_{i}\right)$ plane iff $a \neq 1$.

Proof. Notice that if firm $i$ is selling to shopkeeper $j$, then for any $A_{i} \in \mathbb{R}_{+}, \pi_{i}<a$ if $j=1$ and $\pi_{i}<1$ if $j=2$. Setting $u_{1}^{*}\left(\pi_{i}, A_{i}\right)=K$ and $u_{2}^{*}\left(\pi_{i}, A_{i}\right)=K$ by implicit function theorem we obtain

$$
\frac{d A_{i}}{d \pi_{i}}=\frac{\pi_{i}-a}{2}<0 \text { and } \frac{d A_{i}}{d \pi_{i}}=\frac{\pi_{i}-1}{2}<0 \text { respectively. }
$$


Secondly,

$$
\frac{d^{2} A_{i}}{d \pi_{i}^{2}}=\frac{1}{2}>0 \text { and } \frac{d A_{i}}{d \pi_{i}}=\frac{1}{2}>0 \text { respectively. }
$$

This establishes the convexity property.

Since $u_{j}^{*}\left(\pi_{i}, A_{i}\right)$ is continuous in $\pi_{i}$ and $A_{i}$, for establishing the single crossing property, it suffices to show that for any given pair $\left(\pi_{i}, A_{i}\right), \frac{d A_{i}}{d \pi_{i}}$ for shopkeeper 1 is different from that for shopkeeper 2 which is trivial whenever $a \neq 1$.

We will assume throughout that $a>1$ implying that 1 is the highdemand shopkeeper in our environment. Suppose for a moment that $i$ is a monopolist and assume that $i$ cannot price discriminate. Then, $i$ has two options: (I) either to serve only shopkeeper 1 or (II) to serve both 1 and $2 .^{3}$

Problem (I) Suppose therefore that $i$ serves only 1. Given any pair

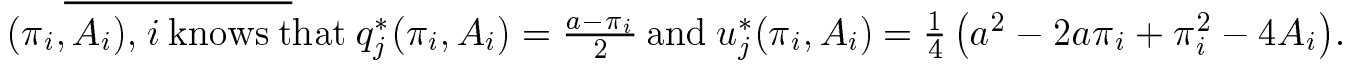
So $i$ solves the following problem.

$$
\begin{array}{cc} 
& \underset{\pi_{i}, A_{i}}{\operatorname{Max}} \pi_{i}\left(\frac{a-\pi_{i}}{2}\right)+A_{i} \\
\text { s.t. }: & \frac{1}{4}\left(a^{2}-2 a \pi_{i}+\pi_{i}^{2}-4 A_{i}\right)=0 .
\end{array}
$$

Thus $i$ 's problem reduces to

$$
\operatorname{Max}_{\pi_{i}}\left(\frac{a-\pi_{i}}{2}\right)+\frac{a^{2}-2 a \pi_{i}+\pi_{i}^{2}}{4}
$$

for which optimality requires

$$
\left(\frac{a-\pi_{i}}{2}\right)-\frac{a}{2}=0
$$

which implies

$$
\pi^{m}(1)=0 \text { and } A^{m}(1)=\frac{a^{2}}{4}
$$

\footnotetext{
${ }^{3}$ Notice that given any $\left(\pi_{i}, A_{i}\right)$, if $u_{2}^{*}\left(\pi_{i}, A_{i}\right) \geq 0$ then it must be that $u_{1}^{*}\left(\pi_{i}, A_{i}\right)>0$. Thus in a monopoly situation, $i$ cannot serve 2 only.
} 
Problem (II) On the other hand suppose $i$ serves both 1 and 2.

Then $i$ 's problem is

$$
\begin{aligned}
& \underset{\pi_{i}, A_{i}}{\operatorname{Max} 2 A_{i}+\pi_{i}}\left(\frac{a+1-2 \pi_{i}}{2}\right) \\
\text { s.t. }: & \frac{1}{4}\left(1-2 \pi_{i}+\pi_{i}^{2}-4 A_{i}\right)=0 .
\end{aligned}
$$

Thus $i$ 's problem reduces to

$$
\underset{\pi_{i}}{\operatorname{Max}} \frac{1-2 \pi_{i}+\pi_{i}^{2}}{2}+\pi_{i}\left(\frac{a+1-2 \pi_{i}}{2}\right) .
$$

Optimality requires

$$
a+1-2 \pi_{i}-2=0
$$

from which we get

$$
\pi^{m}(1,2)=\frac{a-1}{2} \text { and } A^{m}(1,2)=\frac{a^{2}-6 a+9}{16} .
$$

Figure (1) depicts the optimal prices charged by a monopolist. The monopolist's equilibrium will be useful in the remaining of the analysis. Notice that monopoly equilibrium remains unconstrained if and only if $a<3$ as otherwise $\pi^{m}(1,2)$ exceeds 1 for which shopkeeper 2 never buys (i.e. $q_{2}^{*}\left(\pi^{m}(1,2)\right)=0$ whenever $\pi^{m}(1,2) \geq 1$.)

In the following theorem we show that with duopoly, both firms can earn positive profits in equilibrium and $x$ sets a positive royalty while $y$ does not.

Theorem 2 There exists a unique SPE with $\pi_{x} \in(0,1)$ and $\pi_{y}=0$ iff $a \geq 1+\sqrt{2}$. In particular, $\pi_{x}=\frac{1}{\sqrt{2}}<\pi^{m}(1,2)$. Moreover (i) both firms and shopkeeper 1 earn positive payoffs while shopkeeper 2's profit is zero and (ii) $q_{1}^{*}=p_{1}^{*}=a / 2$ and $q_{2}^{*}=\frac{1}{2}-\frac{1}{2 \sqrt{2}}$ and $p_{2}^{*}=\frac{1}{2}+\frac{1}{2 \sqrt{2}}$, implying that the high-demand shopkeeper charges a higher price per copy and sells a higher demand than its low-demand counterpart.

Proof. Suppose $x$ chooses to serve only 1 . Then $x$ knows that when $y$ 's turn comes, $y$ will have the option of serving only 1 or both. If $y$ chooses 


\section{A}

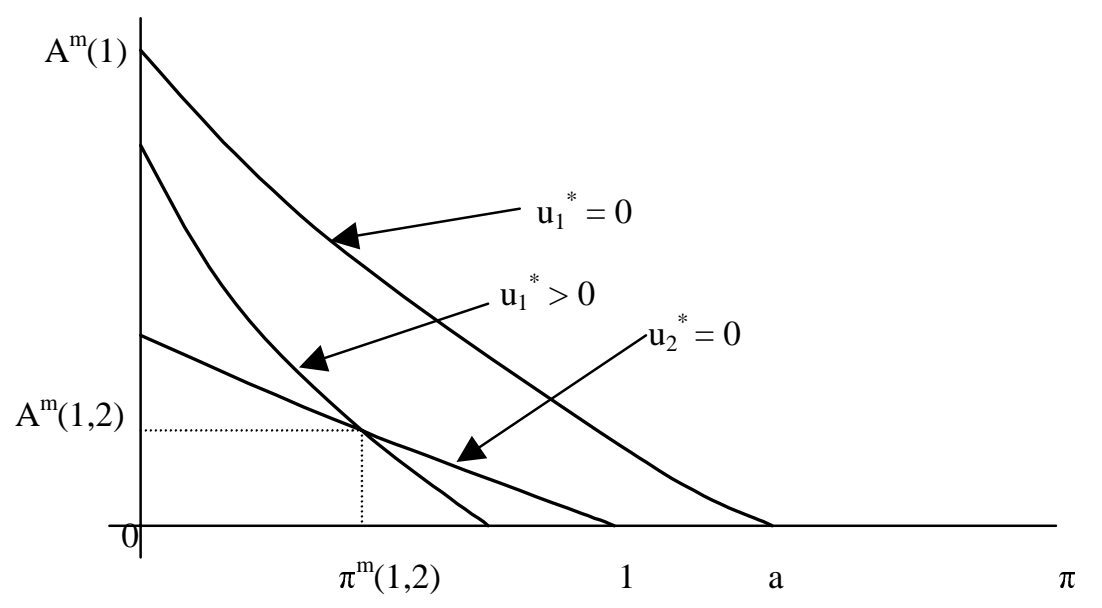

Figure 1: Monopoly Equilibrium

to serve only 1 then $y$ will undercut over $x$ 's strategy. On the other hand if $y$ chooses to serve both clearly 1 will prefer $y$ 's offer to that of $x$ while 2 would remain indifferent between the two offers. With the tie breaking assumption (that whenever a shopkeeper is indifferent between buying from $x$ and $y$ they always buy from $y$ ) $x$ will never offer any price to serve only 1. This would imply that for any $\left(\pi_{x}, A_{x}\right)$ we have $u_{2}^{*}\left(\pi_{x}, A_{x}\right) \geq 0$.

So take any contract $\left(\pi_{x}, A_{x}\right)$ such that $u_{2}^{*}\left(\pi_{x}, A_{x}\right) \geq 0$. Then $y$ has two options: (i) offer $\left(\pi_{y}, A_{y}\right)$ such that only 1 buys or (ii) offer $\left(\pi_{y}, A_{y}\right)$ such that both 1 and 2 buy. Under (i), $y$ 's best strategy, given problem (I) of the monopolist, is to set $\hat{\pi}_{y}=0$ and $\hat{A}_{y}$ such that

$$
\hat{A}_{y}=\frac{2 a \pi_{x}-\pi_{x}^{2}+4 A_{x}}{4} .
$$

Under (ii), $y$ faces the following problem.

$$
\underset{\pi_{y}, A_{y}}{\operatorname{Max}} 2 A_{y}+\pi_{y}\left(\frac{a+1-2 \pi_{y}}{2}\right)
$$

$$
\text { s.t. : } \quad \text { (i) } u_{2}^{*}\left(\pi_{y}, A_{y}\right)=u_{2}^{*}\left(\pi_{x}, A_{x}\right) \text { and (ii) } \pi_{y} \leq \pi_{x} \text {. }
$$


Notice that the above problem is equivalent to problem (II) of the monopolist excepting the fact that now $y$ faces an additional constraint (ii). Since the solution for $\pi$ in the problem (II) of the monopolist is independent of $A$, it follows that the optimal solution of $y$ is

$$
\tilde{\pi}_{y}=\min \left\{\frac{a-1}{2}, \pi_{x}\right\} \text { for any } \pi_{x} \text {, and } \tilde{A}_{y}=\frac{2 \pi_{x}-\pi_{x}^{2}-2 \tilde{\pi}_{y}+\tilde{\pi}_{y}^{2}}{4}+A_{x} .
$$

Given the above, $x$ knows that the only possibility for it to earn a positive profit is to serve 2 and ensure that $y$ serves only 1 . Thus, the problem faced by $x$ is

$$
\begin{array}{ll} 
& \underset{\pi_{x}, A_{x}}{\operatorname{Max}} A_{x}+\pi_{x}\left(\frac{1-\pi_{x}}{2}\right) \\
\text { s.t. } & \text { : (i) } \hat{A}_{y} \geq 2 \tilde{A}_{y}+\tilde{\pi}_{y}\left(\frac{a+1-2 \tilde{\pi}_{y}}{2}\right) \text { and (ii) } u_{2}^{*}\left(\pi_{x}, A_{x}\right) \geq 0 .
\end{array}
$$

Notice that $x$ can always do better by choosing $\left(\pi_{x}, A_{x}\right)$ such that $\hat{A}_{y}=$ $2 \tilde{A}_{y}+\tilde{\pi}_{y}\left(\frac{a+1-2 \tilde{\pi}_{y}}{2}\right)$. Then we get

$$
A_{x}=\frac{a \pi_{x}}{2}+\frac{\pi_{x}^{2}}{4}-\frac{a \tilde{\pi}_{y}}{2}+\frac{\tilde{\pi}_{y}}{2}+\frac{\tilde{\pi}_{y}^{2}}{2}-\pi_{x} .
$$

By successive replacements, $x$ 's problem reduces to

$$
\begin{aligned}
& \underset{\pi_{x}, A_{x}}{\operatorname{Max}} \frac{1}{2} a \pi_{x}-\frac{1}{4} \pi_{x}^{2}-\frac{1}{2} \pi_{y} a+\frac{1}{2} \pi_{y}+\frac{1}{2} \pi_{y}^{2}-\frac{1}{2} \pi_{x} \\
& \text { s.t. }: \quad \frac{1-2 \pi_{x}+\pi_{x}^{2}}{4}-A_{x}=\frac{1}{4}+\frac{1}{2} \pi_{x}-\frac{1}{2} a \pi_{x}+\frac{1}{2} \pi_{y} a-\frac{1}{2} \pi_{y}-\frac{1}{2} \pi_{y}^{2} \geq 0 .
\end{aligned}
$$

Suppose $\pi_{x} \leq \frac{a-1}{2}$. Then, $\tilde{\pi}_{y}=\pi_{x}$. Then $x$ 's problem reduces to

$$
\begin{array}{cc} 
& \operatorname{Max}_{\pi_{x}} \frac{1}{4} \pi_{x}^{2} \\
\text { s.t. } \quad: \quad & \frac{1}{4}-\frac{1}{2} \pi_{x}^{2} \geq 0 .
\end{array}
$$

The unique solution to the above problem is

$$
\pi_{x}^{*}=\frac{1}{\sqrt{2}} \text { and } A_{x}^{*}=\frac{3}{8}-\frac{1}{2 \sqrt{2}} \in(0,1) .
$$


Then,

$$
\hat{A}_{y}=\frac{a}{2 \sqrt{2}}+\frac{1}{4}-\frac{1}{\sqrt{2}} .
$$

Thus the payoffs of the players are:

$$
\begin{aligned}
U_{x}^{*}\left(\pi_{x}^{*}, A_{x}^{*}, \hat{\pi}_{y}, \hat{A}_{y}\right) & =\frac{1}{8}>0, U_{y}^{*}\left(\pi_{x}^{*}, A_{x}^{*}, \hat{\pi}_{y}, \hat{A}_{y}\right)=\frac{a}{2 \sqrt{2}}+\frac{1}{4}-\frac{1}{\sqrt{2}} \text { and } \\
u_{1}^{*}\left(\pi_{x}^{*}, A_{x}^{*}, \hat{\pi}_{y}, \hat{A}_{y}\right) & =\frac{a^{2}}{4}-\frac{a}{2 \sqrt{2}}-\frac{1}{4}+\frac{1}{\sqrt{2}}>0, \\
u_{2}^{*}\left(\pi_{x}^{*}, A_{x}^{*}, \hat{\pi}_{y}, \hat{A}_{y}\right) & =\frac{1-2 \frac{1}{\sqrt{2}}+\frac{1}{2}}{4}-\left(\frac{3}{8}-\frac{1}{4} \sqrt{2}\right)=0 .
\end{aligned}
$$

Now, notice that we started with the assumption that $\pi_{x}^{*} \leq \frac{a-1}{2}$. But this implies that $a \geq 1+\sqrt{2}$. Now at $a=1+\sqrt{2}$ we have

$$
U_{y}^{*}\left(\pi_{x}^{*}, A_{x}^{*}, \hat{\pi}_{y}, \hat{A}_{y}\right)=\frac{3}{4}-\frac{1}{4} \sqrt{2}>0,
$$

which implies that in the relevant range of $\pi_{x}^{*} \leq \frac{a-1}{2}$, we have

$$
U_{y}^{*}\left(\pi_{x}^{*}, A_{x}^{*}, \hat{\pi}_{y}, \hat{A}_{y}\right)=\frac{a}{2 \sqrt{2}}+\frac{1}{4}-\frac{1}{\sqrt{2}}>0
$$

Now suppose $\pi_{x}>\frac{a-1}{2}$. Take any $\left(\pi_{x}, A_{x}\right)$. If $y$ wants to undercut $x$ and serve both shopkeepers, then $y$ offers $\left(\tilde{\pi}_{y}, \tilde{A}_{y}\right)$ such that $u_{2}^{*}\left(\tilde{\pi}_{y}, \tilde{A}_{y}\right)=$ $u_{2}^{*}\left(\pi_{x}, A_{x}\right)$. Given problem (II) of the monopolist, we have

$$
\left(\tilde{\pi}_{y}, \tilde{A}_{y}\right)=\left(\frac{a-1}{2}, \frac{2-a}{4}+\frac{(a-1)^{2}}{16}+\frac{2 \pi_{x}-\pi_{x}^{2}-1}{4}+A_{x}\right) .
$$

On the other hand, if $y$ wants to serve only 1 by the strategy $\left(\hat{\pi}_{y}, \hat{A}_{y}\right)$ then given $\left(\pi_{x}, A_{x}\right)$ and problem (I) of the monopolist, it must be that $\hat{\pi}_{y}=0$ and $u_{1}^{*}\left(0, \hat{A}_{y}\right)=u_{1}^{*}\left(\pi_{x}, A_{x}\right)$. From this we obtain

$$
\hat{A}_{y}=A_{x}+\frac{2 a \pi_{x}-\pi_{x}^{2}}{4} .
$$

Thus $x$ solves the following problem.

$$
\begin{gathered}
\underset{\pi_{x}, A_{x}}{\operatorname{Max}} A_{x}+\pi_{x}\left(\frac{1-\pi_{x}}{2}\right) \\
\text { s.t. }: \quad \text { (i) } \hat{A}_{y}=\tilde{A}_{y}+\tilde{\pi}_{y}\left(\frac{a-\tilde{\pi}_{y}}{2}\right) \text { and (ii) } u_{2}^{*}\left(\pi_{x}, A_{x}\right) \geq 0 .
\end{gathered}
$$




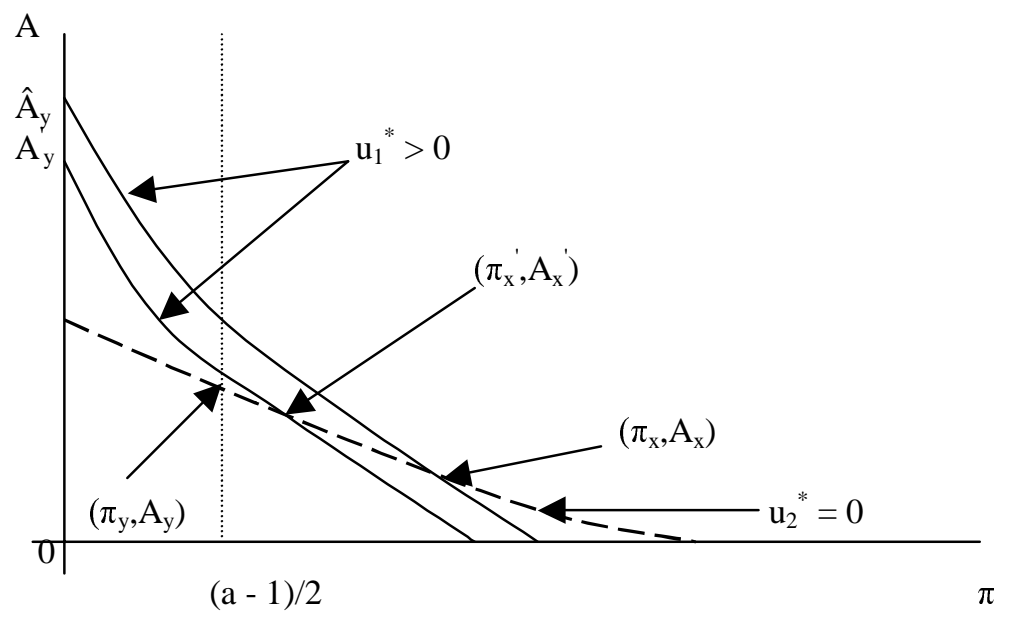

Figure 2: Nonexistence of subgame perfect equilibria with $\pi_{x}>\frac{a-1}{2}$.

Now, from constraint (i) we obtain

$$
\pi_{x}=\frac{3(a-1)}{8} .
$$

Thus it must be that $\pi_{x}^{*}=\frac{3(a-1)}{8}$. But since we assumed that $\pi_{x}^{*}>\frac{a-1}{2}$, the solution is impossible. This implies that $\pi_{x}^{*} \leq \frac{a-1}{2}$ and therefore $\tilde{\pi}_{y}=\pi_{x}^{*}$ and we are back in (i). Consequently the shopkeepers profit maximizing strategies are $q_{1}^{*}=p_{1}^{*}=a / 2$ and $q_{2}^{*}=\frac{1}{2}-\frac{1}{2 \sqrt{2}}$ and $p_{2}^{*}=\frac{1}{2}+\frac{1}{2 \sqrt{2}}$.

Consider figure 2 which assumes that $\pi_{x}^{*}>\frac{a-1}{2}$.

Suppose $x$ chooses some $\left(\pi_{x}, A_{x}\right)$. Then it is clear that if $y$ wants to undercut and take both the shopkeepers, then $y$ 's best strategy is to set $\pi_{y}=\frac{a-1}{2}$ and offer a consequent fixed price equal . Such a pricing contract from $y$ is the point $\left(\pi_{y}, A_{y}\right)$. On the other hand given $\left(\pi_{x}, A_{x}\right)$ if $y$ wants to serve only shopkeeper 1 , then its best strategy is to set $\pi_{y}=0$ and $A_{y}=$ $\hat{A}_{y}$. Suppose for the moment that $y$ 's payoff between $\left(\pi_{y}, A_{y}\right)$ and $\left(0, \hat{A}_{y}\right)$ is equal. Then we showed in part (ii) of the proof of theorem 2 that $\pi_{x}$ 
cannot be at the same time greater than $\frac{a-1}{2}$. Thus the only other solution possible is when $x$ designs its offer such that $y$ receives a payoff out of $\left(0, \hat{A}_{y}\right)$ strictly greater than what it receives under $\left(\pi_{y}, A_{y}\right)$. So suppose that $y$ 's payoff under $\left(0, \hat{A}_{y}\right)$ is strictly greater than under $\left(\pi_{y}, A_{y}\right)$. Given this, $x$ would find it optimal to move to a new strategy $\left(\pi_{x}^{\prime}, A_{x}^{\prime}\right)$ which is to the left (along the $u_{2}^{*}\left(\left(\pi_{x}, A_{x}\right)\right.$ locus) of $\left(\pi_{x}, A_{x}\right)$ as firms always prefer to reduce the royalty and increase the fixed price for any given level of shopkeeper's utility (this follows directly from problem (I) of the monopolist). In fact, $x$ 's optimal choice of $\left(\pi_{x}^{\prime}, A_{x}^{\prime}\right)$ would be exactly where given $\left(\pi_{x}^{\prime}, A_{x}^{\prime}\right), y$ remains indifferent between serving both the consumers (by offering $\left.\left(\pi_{y}, A_{y}\right)\right)$ and serving only 1 by offering $\left(0, A_{y}\right)$. But this brings us back to the situation where given the contract offered by $x, y$ becomes indifferent between serving only 1 and serving both, a case where a solution cannot exist if we also require $\pi_{x}$ to be strictly greater than $\frac{a-1}{2}$.

Now suppose we allow $\pi_{x} \leq \frac{a-1}{2}$. Then from the proof of theorem 2 we find that there exists a unique subgame perfect equilibrium that is depicted in figure 3. Notice that the equilibrium strategy of $x$ is independent of $a$, the demand intercept of the high demand shopkeeper. On the other hand, as $a$ increases, the equilibrium fixed price charged by $y$ increases affinely. For example in the figure, $1+\sqrt{2}<a_{1}<a_{2}$ and thus $\hat{A}_{y}^{*}\left(a_{1}\right)<$ $\hat{A}_{y}^{*}\left(a_{2}\right)$. Moreover, such a monotonic divergence is also obtained between the prices charged by the shopkeepers. In particular, as $a$ increases, the price charged by shopkeeper 1 increases strictly while that of shopkeeper 2 remains constant.

The intuition of the above result is as follows. When setting a price strategy, $x$ realizes that since $y$ can always price undercut and take away the entire demand from $x$, the only way $x$ can remain in business with strictly positive profit is by forcing $y$ not to serve at least one shopkeeper. However, $x$ also knows that whenever the low demand user buys, the high demand user will also buy. Thus, $x$ knows that it will earn positive profit if and only if $y$ serves only to high demand users. Can $x$ find a pricing strategy such that it is in the best interest for $y$ itself to design a package such that 


\section{A}

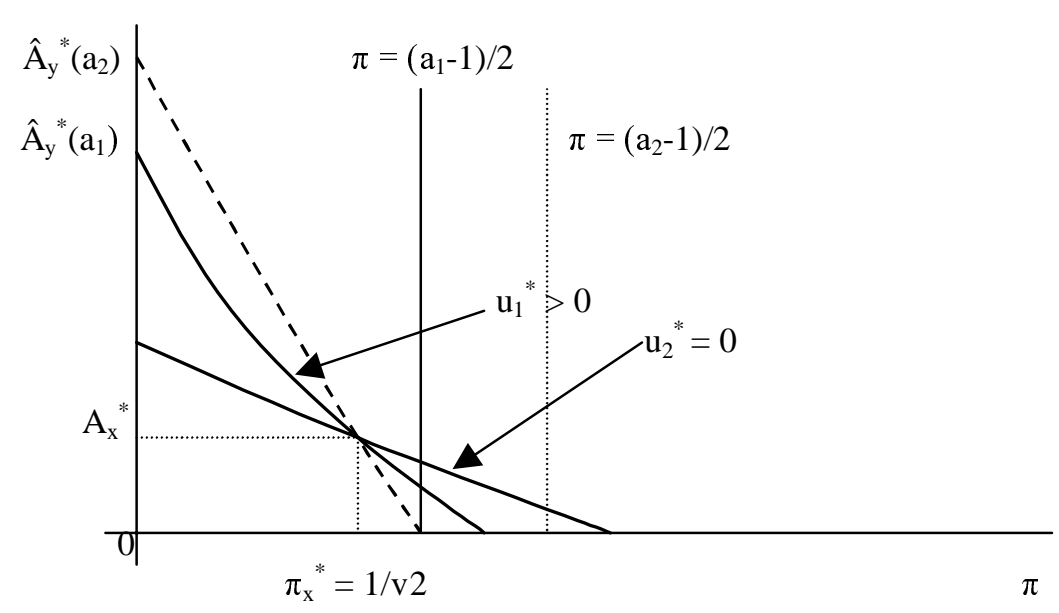

Figure 3: In the unique subgame perfect equilibrium $x$ sets $\pi_{x}=\frac{1}{\sqrt{2}}<$ $\pi^{m}(1,2)$ and serves the low demand shopkeeper while $y$ sets $\pi_{y}=0$ and $\hat{A}_{y}^{*}=\frac{a}{2 \sqrt{2}}+\left(\frac{1}{4}-\frac{1}{\sqrt{2}}\right)$ and serves the high demand one. 
high demand user prefers $y$ 's package to that of $x$ while the low demand user prefers $x$ 's package to that of $y$. But this is possible only if $x$ 's package itself is such that undercutting it and selling to both shopkeepers yields very low profits. Precisely why $x$ sets such a low fixed price and royalty, making $y$ instead offer only a fixed price and target the high demand user.

\section{Concluding remarks}

In a simple setting we have shown that even in a non-repeated environment, Xerox (the incumbent firm) and Canon (the entrant) would find it optimal not to enter in a price war but rather segment the market into individual monopolies. Evidently in our model Canon has a second-mover advantage as expected in a sequential pricing game. In reality however, there are other reasons for avoiding price wars. For example, the machines sold by the firms may not in the first place be homogenous. In that case the valuations of the shopkeepers may differ and implicitly segment the market, giving room to the firms to earn positive profits with price competition. Secondly, threat of future undercutting may itself lead to some form of tacit collusion where each firm finds it optimal to serve only a particular type of buyers. What we show is that even in the absence of product differentiation and tacit collusion, it may be in the best interest of the firms not to enter price wars.

\section{References}

[1] Bertrand, J. (1883). "Théorie mathématique de la richesse sociale", $J$. Savants, pp 499-508.

[2] Blackstone, E. (1975). "Restrictive practices in the marketing of electrofax copying machines and supplies: the SCM corporation case", Journal of Industrial Economics, 23, pp. 189-202.

[3] Ireland, N. J. (1991). "Welfare and non-linear pricing in a Cournot oligopoly", The Economic Journal, 101, pp. 949-957. 
[4] Kreps, D. and J. Scheinkman. (1983). "Quantity pre-commitment and Bertrand competition yield Cournot outcomes", Bell Journal of Economics, 14, pp. 326-337.

[5] Morgan, P. B. and O. Shy (1996). "Undercut-proof equilibria", Tel Aviv University, Foerder Institute for Economic Research Working Paper: 20/96, July.

[6] Mussa, R. and S. Rosen (1978). "Monopoly and product quality", Journal of Economic Theory, 18, pp 301-317.

[7] Spence, M. (1977). "Non-linear prices and welfare", Journal of Public Economics, 8, pp. 1-18.

[8] Tirole, J. (1994). "The theory of Industrial Organization", MIT Press, Cambridge, Massa chusetts.

[9] Wallner, K. (1999). "Sequential moves and tacit collusion: reactionfunction cycles in a finite pricing duopoly", Journal of Economic Theory, 84, pp. 251-267. 